\title{
The roll-up of vorticity strips on the surface of a sphere
}

\author{
By DAVID G. DRITSCHEL $L^{1}$ AND LORENZO M. POLVANI ${ }^{2}$ \\ ${ }^{1}$ Department of Applied Mathematics and Theoretical Physics, University of Cambridge, \\ Silver Street, Cambridge CB3 9EW, UK \\ ${ }^{2}$ Department of Applied Physies, Columbia University, New York, NY 10027, USA
}

(Received 12 December 1990)

We derive the conditions for the stability of strips or filaments of vorticity on the surface of a sphere. We find that the spherical results are surprisingly different from the planar ones, owing to the nature of the spherical geometry. Strips of vorticity on the surface of a sphere show a greater tendency to roll-up into vortices than do strips on a planar surface.

The results are obtained by performing a linear stability analysis of the simplest, piecewise-constant vorticity configuration, namely a zonal band of uniform vorticity located in equilibrium between two latitudes. The presence of polar vortices is also considered, this having the effect of introducing adverse shear, a known stabilizing mechanism for planar flows. In several representative examples, the fully developed stages of the instabilities are illustrated by direct numerical simulation.

The implication for planetary atmospheres is that barotropic flows on the sphere have a more pronounced tendency to produce small, long-lived vortices, especially in equatorial and mid-latitude regions, than was previously anticipated from the theoretical results for planar flows. Essentially, the curvature of the sphere's surface weakens the interaction between different parts of the flow, enabling these parts to behave in relative isolation.

\section{Introduction}

The problem of the stability of a strip of uniform vorticity was originated by Rayleigh (1887), and the finding most frequently quoted - that the strip is maximally unstable to disturbances having a wavelength about eight times the width of the strip - has been applied numerous times to explain observational, experimental, and numerical results. A finding less well known, largely because Rayleigh did not give explicit details, is that adverse shear can prevent this instability (see Dritschel $1989 a$ ). In this paper we study how these classical results carry over to flows on the surface of a sphere.

This subject is of fundamental relevance to the dynamics of the Earth's stratosphere, particularly to the mixing of chemical tracers and the impact this has on ozone depletion (McIntyre 1991). However it also addresses the nature of the circulation itself, that is, whether the circulation is characterized by a dominant vortex plus quasi-passive filament dynamics, a population of small-scale vortices scattered around the polar vortex, or a combination of the two. At present, observational data are not sufficiently dense and accurate to visualize (potential) vorticity structures on scales significantly smaller than the polar vortex.

Our interest in this problem was generated by some recent numerical experiments 
of barotropic flow on the surface of a sphere (Juckes 1987 ; Juckes \& McIntyre 1987). Using a hemispherical model, they examined the response of a broadly distributed, initially zonal vortex centred at the pole to large-scale forcing, such as might result in the wintertime stratosphere from tropospheric disturbances below. They observed both the development of a sharp vortex edge and, more surprisingly, the roll-up of secondary vortices from ejected strips of vorticity equatorward of the main polar vortex. This roll-up cannot be reconciled with the results of strip stability in the planar case, which suggest that the adverse shear induced by the polar vortex should make vorticity strips stable (Dritschel $1989 a$ ).

To resolve this difficulty we examine the stability of the simplest relevant vorticity configuration on the surface of a sphere. A strip of uniform vorticity spanning a band of latitudes is subjected to small-amplitude disturbances. By a linear eigenvalue analysis $(\$ \S 2-3)$, we recover the conditions for stability and the dispersion relation, as a function of three essential parameters: the equilibrium latitude of the northern and southern edges of the strip, and the difference in the vorticity jumps across the two edges. Several of the more unusual instabilities are followed deep into their nonlinear stages using a contour dynamics algorithm. In $\$ 4$, the effects of a single polar vortex or a pair of polar vortices (one at each pole) are examined, and general conditions for roll-up established. We find that when a thin vorticity strip is sufficiently far from the polar region, the adverse shear due to the polar vortex is not sufficient to make it stable. This may in some part account for the roll-up observed in the stratospheric simulations of Juckes (1987), Juckes \& McIntyre (1987) and Waugh (1991), and could account for the near-equatorial location of large vortices on the outer planets. A simple explanation of the observed instability is given in $\$ 5$, and the findings are summarized in $\S 6$.

\section{Mathematical formulation}

In this section, we review the governing equations for inviscid, incompressible flow on a sphere and derive several results useful for the linear analyses in $\$ \$ 3-4$.

The equations of motion for two-dimensional flow on the surface of a sphere of radius 1 can be written in the form

$$
\mathrm{d} x / \mathrm{d} t=\boldsymbol{u}(\boldsymbol{x})=-\frac{1}{4 \pi} \sum_{k} \tilde{\omega}_{k} \oint_{\mathscr{C}_{k}} \log \left|x-X_{k}\right|^{2} \mathrm{~d} X_{k}
$$

(Dritschel 1988a; for a brief derivation, see Appendix A), provided that the vorticity distribution is piecewise constant, with the vorticity jumping by the values $\tilde{\omega}_{k}$ across the contours $\mathscr{C}_{k}$. This assumption may seem severe, but in practice a modest number of contours can well approximate the dynamics of a continuous vorticity distribution (Legras \& Dritschel 1991). From (1) the Lagrangian motion of a fluid particle at any point $\boldsymbol{x}$ can be computed as a sum of contour integrals. Since (1) applies as well to the points located on the contours $\mathscr{C}_{k}$, it determines the evolution of the system completely.

Note that a spherical fluid shell rotating as a solid body, such as a rigidly rotating atmosphere, is not the most convenient flow to be modelled in this way, since it corresponds to a continuous vorticity gradient (a linear function of the axial coordinate $z$ ) which would have to be approximated by a series of discrete steps separating regions of piecewise uniform vorticity. However, recent observations of the actual (absolute, potential) vorticity distribution of the Earth's stratosphere on isentropic surfaces show that it is, in fact, quite far from rigid rotation (Juckes \& 


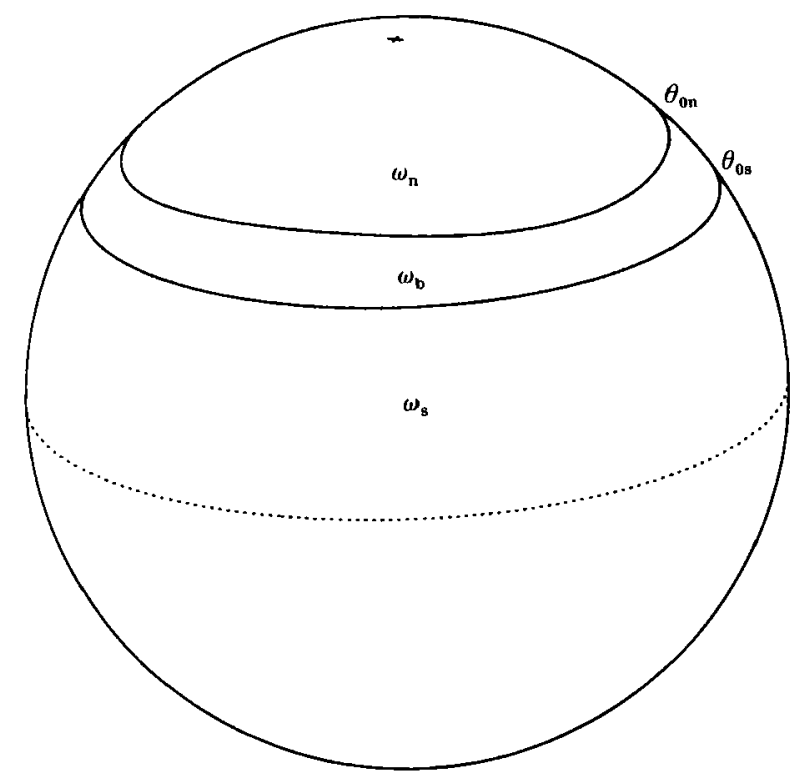

Figure 1. A sketch of the basic flow geometry. A band of vorticity $\omega=\omega_{\mathrm{b}}$ lies between the latitudes $\theta=\theta_{0 \mathrm{~s}}$ and $\theta_{0 \mathrm{n}}$. To the south of the band, $\omega=\omega_{\mathrm{s}}$, and to the north, $\omega=\omega_{\mathrm{n}}$. The dashed contour is the equator, and the small cross shows the position of the north pole.

McIntyre 1987) and much closer to a piecewise uniform distribution, owing to the continual sharpening of vorticity gradients by dynamical effects (Dritschel \& Legras 1991).

The reason for using (1) is two-fold. First, there is a perfect symmetry among the three Cartesian coordinates, something which is not apparent in the common formulation in latitude-longitude variables. Second, there is no need to refer to stream function and pressure when deriving the linear stability equations since all of the dynamies are incorporated in (1).

Consider first the equilibrium vorticity profile illustrated in figure 1 . A band of vorticity $\omega_{\mathrm{b}}$ lies between the latitudes $\theta_{\mathrm{os}}$ and $\theta_{0 \mathrm{n}}$. To the north, the vorticity is $\omega_{\mathrm{n}}$, while to the south it is $\omega_{\mathrm{s}}$. This profile is more general than the one first considered by Rayleigh for planar flow, that of a strip of vorticity embedded in irrotational flow, but similar to the one he considered subsequently. Because the total circulation on a finite surface must vanish (the surface integral of Laplace's equation is zero), the three vorticity values $\omega_{\mathrm{n}}, \omega_{\mathrm{b}}$, and $\omega_{\mathrm{s}}$ are not independent. Using the relation between axial coordinate and latitude (for a sphere of unit radius), $z=\sin \theta$, the constraint of zero total circulation requires

$$
\left(1-z_{0 \mathrm{n}}\right) \omega_{\mathrm{n}}+\left(z_{0 \mathrm{n}}-z_{0 \mathrm{~s}}\right) \omega_{\mathrm{b}}+\left(z_{0 \mathrm{~s}}+1\right) \omega_{\mathrm{s}}=0 .
$$

In fact, (1) implicitly incorporates this constraint, and this is why only the jumps in vorticity across contours appear in (1) (the number of uniform-vorticity regions is always one more than the number of jumps). The jumps across the northern and southern contours bounding the strip are

$$
\tilde{\omega}_{\mathrm{n}}=\omega_{\mathrm{n}}-\omega_{\mathrm{b}}, \quad \tilde{\omega}_{\mathrm{s}}=\omega_{\mathrm{b}}-\omega_{\mathrm{s}}
$$

and with these values, one may compute the basic flow from (1), albeit laboriously. 
Since the basic flow is zonally symmetric, it is easier to work with the equation relating the zonal velocity $U$ to the vorticity $\omega$ :

$$
-\frac{1}{\cos \theta} \frac{\mathrm{d}}{\mathrm{d} \theta}(U \cos \theta)=\omega .
$$

The quantity most useful for the stability analysis is not in fact $U$ but rather the angular velocity $\Omega=U / \cos \theta$. It is also more convenient to work with the axial coordinate $z$ in place of latitude $\theta$. In the $z$ variable, $\Omega$ is determined from

$$
-\mathrm{d} / \mathrm{d} z\left[\left(1-z^{2}\right) \Omega\right]=\omega .
$$

Integrating this equation, it is easy to show that the angular velocities at the two edges of the undisturbed band of vorticity are

$$
\Omega_{0 \mathrm{n}}=\frac{\omega_{\mathrm{n}}}{1+z_{0 \mathrm{n}}}, \quad \Omega_{0 \mathrm{~s}}=-\frac{\omega_{\mathrm{s}}}{1-z_{0 \mathrm{~s}}} .
$$

Consider next disturbing the edges of the strip. The $z$ position of the two edges are denoted $z_{\mathrm{n}}(\phi, t)$ and $z_{\mathrm{s}}(\phi, t)$, where $\phi$ is longitude. Expanding about the basic state, we put

$$
z_{\mathrm{n}, \mathrm{s}}(\phi, t)=z_{0 \mathrm{n}, \mathrm{s}}+\zeta_{\mathrm{n}, \mathrm{s}}(\phi, t),
$$

where $\zeta_{n, s} \ll 1$. The other coordinates along the contour are disturbed as a consequence of $(7)$, since $x^{2}+y^{2}+z^{2}=1$. To obtain the linear equations satisfied by $\zeta_{n, s}$, however, it is sufficient to substitute (7) into the $z$-component of (1). This gives

$$
\begin{array}{r}
\frac{\mathrm{d} \zeta_{\mathrm{n}}}{\mathrm{d} t}=\frac{\partial \zeta_{\mathrm{n}}}{\partial t}+\Omega_{\mathrm{on}} \frac{\partial \zeta_{\mathrm{n}}}{\partial \phi}=-\frac{\tilde{\omega}_{\mathrm{n}}}{4 \pi} \int_{0}^{2 \pi} \log \left|x_{0 \mathrm{n}}(\alpha)-x_{0 \mathrm{n}}(\phi)\right|^{2} \frac{\partial \zeta_{\mathrm{n}}}{\partial \alpha} \mathrm{d} \alpha \\
-\frac{\tilde{\omega}_{\mathrm{s}}}{4 \pi} \int_{0}^{2 \pi} \log \left|x_{\mathrm{os}}(\alpha)-x_{\mathrm{on}}(\phi)\right|^{2} \frac{\partial \zeta_{\mathrm{s}}}{\partial \alpha} \mathrm{d} \alpha, \\
\frac{\mathrm{d} \zeta_{\mathrm{s}}}{\mathrm{d} t}=\frac{\partial \zeta_{\mathrm{s}}}{\partial t}+\Omega_{\mathrm{os}} \frac{\partial \zeta_{\mathrm{s}}}{\partial \phi}=-\frac{\tilde{\omega}_{\mathrm{n}}}{4 \pi} \int_{0}^{2 \pi} \log \left|x_{\mathrm{on}}(\alpha)-x_{0 \mathrm{~s}}(\phi)\right|^{2} \frac{\partial \zeta_{\mathrm{n}}}{\partial \alpha} \mathrm{d} \alpha \\
-\frac{\tilde{\omega}_{\mathrm{s}}}{4 \pi} \int_{0}^{2 \pi} \log \left|x_{\mathrm{os}}(\alpha)-x_{0 \mathrm{~s}}(\phi)\right|^{2} \frac{\partial \zeta_{\mathrm{s}}}{\partial \alpha} \mathrm{d} \alpha,
\end{array}
$$

where the angular velocities along the undisturbed contours $\Omega_{0 \mathrm{n}, \mathrm{s}}$ are given in (6) above.

The eigensolutions of (8) are found by letting

$$
\zeta_{n, s}=\hat{\zeta}_{n, s} e^{i(m \phi-\sigma t)}+\text { c.c., }
$$

where $m$ is the azimuthal wavenumber and $\sigma$ is the eigenvalue or eigenfrequency, and substituting these expressions into (8) to obtain

$$
\begin{gathered}
\left(m \Omega_{0 \mathrm{n}}-\sigma\right) \hat{\zeta}_{\mathrm{n}}=\frac{1}{2}\left[\tilde{\omega}_{\mathrm{n}} F_{m}\left(z_{0 \mathrm{n}}, z_{0 \mathrm{n}}\right) \hat{\zeta}_{\mathrm{n}}+\tilde{\omega}_{\mathrm{s}} F_{m}\left(z_{0 \mathrm{n}}, z_{0 \mathrm{~s}}\right) \hat{\zeta}_{\mathrm{s}}\right] \\
\left(m \Omega_{0 \mathrm{~s}}-\sigma\right) \hat{\zeta}_{\mathrm{s}}=\frac{1}{2}\left[\tilde{\omega}_{\mathrm{n}} F_{m}\left(z_{0 \mathrm{~s}}, z_{0 \mathrm{n}}\right) \hat{\zeta}_{\mathrm{n}}+\tilde{\omega}_{\mathrm{s}} F_{m}\left(z_{0 \mathrm{~s}}, z_{0 \mathrm{~s}}\right) \hat{\zeta}_{\mathrm{s}}\right]
\end{gathered}
$$

where

$$
\begin{aligned}
F_{m}\left(z_{a}, z_{b}\right) & \equiv-\frac{m}{2 \pi} \int_{0}^{2 \pi} \log \left(1-z_{a} z_{b}-\left(1-z_{a}^{2}\right)^{\frac{1}{2}}\left(1-z_{b}^{2}\right)^{\frac{1}{2}} \cos \beta\right) \mathrm{e}^{\mathrm{i} m \beta} \mathrm{d} \beta \\
& =\left(\frac{1-z_{>}}{1+z_{>}} \frac{1+z_{<}}{1-z_{<}}\right)^{m / 2}
\end{aligned}
$$

and $z_{>}$stands for the maximum of $z_{a}$ and $z_{b}$, while $z_{<}$stands for the minimum. 
We note en passant that had one tried to carry out this linear stability analysis in the traditional way - say, following Kelvin's analysis of the Rankine vortex (Lamb 1932) - one would have had to determine the form of the stream function associated with the boundary disturbance. This stream function must satisfy Laplace's equation on the spherical surface. We have been unable to find any mention of such functions in the literature, and for reference we present them here. The harmonic functions on the spherical surface are $[(1-z) /(1+z)]^{m / 2} \mathrm{e}^{\mathrm{i} m \phi}$ to the north of each contour and $[(1+z) /(1-z)]^{m / 2} \mathrm{e}^{\mathrm{i} m \phi}$ to the south. Owing to the topology of the sphere, functions that are harmonic and well behaved over the whole sphere cannot be found.

There is an obvious generalization of (10) to more than two contours, namely

$$
\left(m \Omega_{0 j}-\sigma\right) \hat{\zeta}_{j}=\frac{1}{2} \sum_{k} \tilde{\omega}_{k} F_{m}\left(z_{0 j}, z_{0 k}\right) \hat{\zeta}_{k}
$$

and the generalization of $(6)$ is

$$
\begin{aligned}
\Omega_{0 j} & =\frac{1}{2} \sum_{k} \tilde{\omega}_{k}\left(\frac{1-z_{0 k}^{2}}{1-z_{0 j}^{2}}\right)^{\frac{1}{2}} F_{1}\left(z_{0 j}, z_{0 k}\right) \\
& =\frac{1}{2}\left\{\sum_{k>j} \tilde{\omega}_{k} \frac{1+z_{0 k}}{1+z_{0 j}}+\tilde{\omega}_{j}+\sum_{k<j} \tilde{\omega}_{k} \frac{1-z_{0 k}}{1-z_{0 j}}\right\},
\end{aligned}
$$

where $z_{01}>z_{02}>\ldots$

\section{Stability results for a band of vorticity}

In this section, we present the eigensolutions of (10) and then illustrate the nonlinear development and saturation of several unstable disturbances by direct numerical simulation.

Noting that $F_{m}=1$ if the arguments are equal, and $F_{m}\left(z_{0 n}, z_{0 \mathrm{~s}}\right)=F_{m}\left(z_{0 \mathrm{~s}}, z_{0 \mathrm{n}}\right)=S^{m}$, where

$$
S \equiv\left(\frac{1-z_{0 \mathrm{n}}}{1+z_{0 \mathrm{n}}} \frac{1+z_{0 \mathrm{~s}}}{1-z_{0 \mathrm{~s}}}\right)^{\frac{1}{2}},
$$

(10) simplifies to

$$
\begin{gathered}
\left(m \Omega_{0 \mathrm{n}}-\sigma-\frac{1}{2} \tilde{\omega}_{\mathrm{n}}\right) \hat{\zeta}_{\mathrm{n}}-\frac{1}{2} \tilde{\omega}_{\mathrm{s}} S^{m} \hat{\zeta}_{\mathrm{s}}=0 \\
-\frac{1}{2} \tilde{\omega}_{\mathrm{n}} S^{m} \hat{\zeta}_{\mathrm{n}}+\left(m \Omega_{\mathrm{os}}-\sigma-\frac{1}{2} \tilde{\omega}_{\mathrm{s}}\right) \hat{\zeta}_{\mathrm{s}}=0
\end{gathered}
$$

Solvability requires that the determinant of (15) be zero, so

$$
\left(m \Omega_{0 \mathrm{n}}-\sigma-\frac{1}{2} \tilde{\omega}_{\mathrm{n}}\right)\left(m \Omega_{0 \mathrm{~s}}-\sigma-\frac{1}{2} \tilde{\omega}_{\mathrm{s}}\right)-\frac{1}{4} \tilde{\omega}_{\mathrm{n}} \tilde{\omega}_{\mathrm{s}} S^{2 m}=0,
$$

whose solution yields the eigenvalue $\sigma$,

$$
\sigma=\frac{1}{2}\left[-\omega_{+} \pm\left(\omega_{-}^{2}+\tilde{\omega}_{\mathrm{n}} \tilde{\omega}_{\mathrm{s}} S^{2 m}\right)^{\frac{1}{2}}\right]
$$

where

$$
\omega_{+} \equiv \frac{1}{2}\left(\tilde{\omega}_{\mathrm{n}}+\tilde{\omega}_{\mathrm{s}}\right)-m\left(\Omega_{0 \mathrm{n}}+\Omega_{0 \mathrm{~s}}\right) \quad \omega_{-} \equiv \frac{1}{2}\left(\tilde{\omega}_{\mathrm{n}}-\tilde{\omega}_{\mathrm{s}}\right)-m\left(\Omega_{0 \mathrm{n}}-\Omega_{0 \mathrm{~s}}\right)
$$

The existence of two roots is a result of there being two contours. The number of eigenvalues is equal to the number of contours in the basic vorticity distribution.

Stability therefore depends on the sign of the term in the radical in $(17 a)$. It is immediately clear that $\tilde{\omega}_{n} \tilde{\omega}_{\mathrm{s}}>0$ is a sufficient condition for stability for all azimuthal wavenumbers $m$. This condition corresponds to a monotonic vorticity 

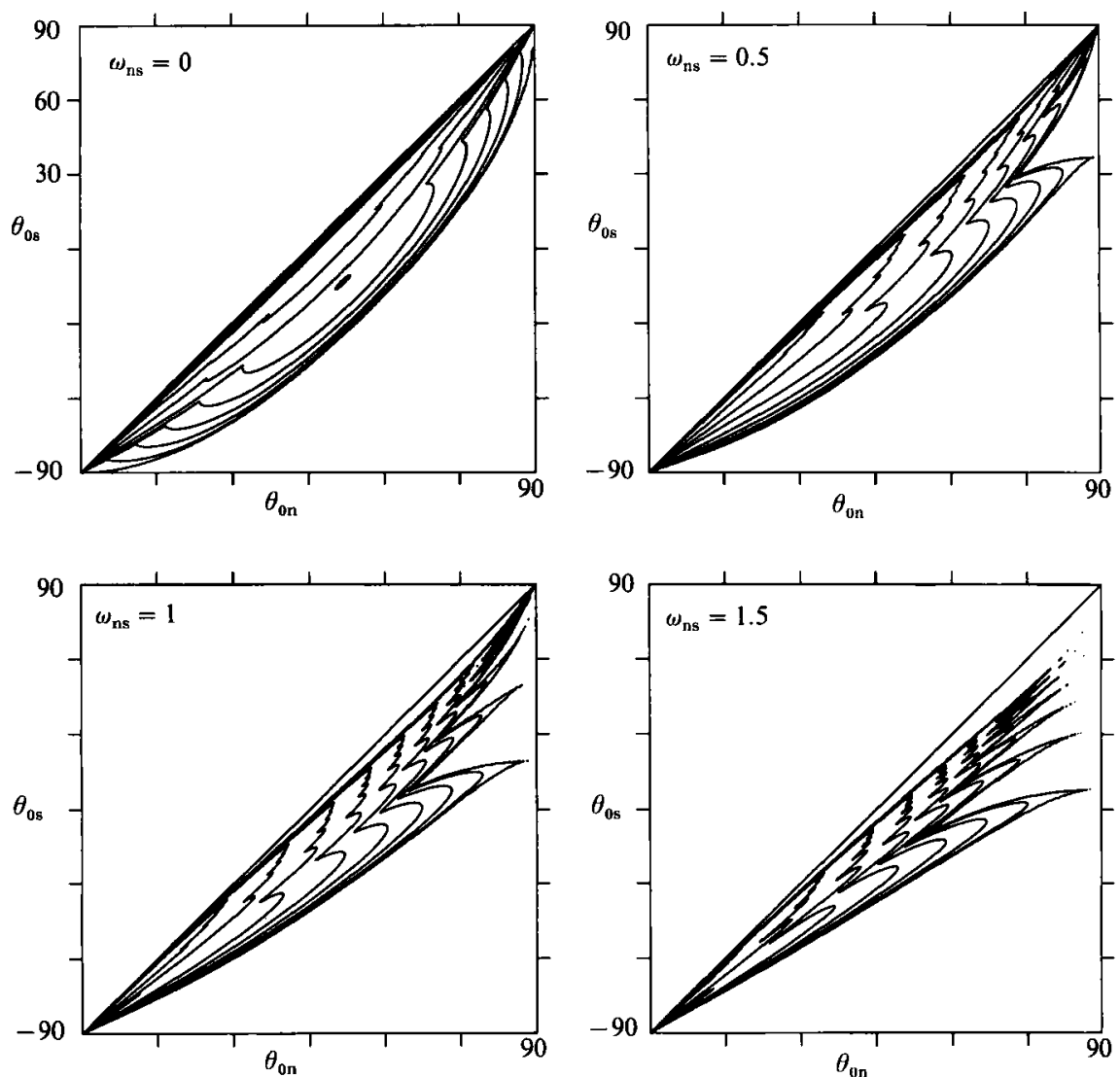

Figure 2, Contour maps of the growth rate $\sigma_{\mathrm{i}}$ maximized over the first eight azimuthal wavenumbers as a function of $\theta_{0 \mathrm{n}}$ and $\theta_{\mathrm{os}}$, for selected values of the north-south vorticity difference, $\omega_{\text {ns }}$. The contour interval is 0.025 , and the outermost contour corresponds to the stability boundary $\left(\sigma_{1}=0\right)$.

distribution. In fact, under this condition, the flow is stable to finite-amplitude disturbances, in the sense that

$$
\tilde{\omega}_{\mathrm{n}} \oint_{\mathscr{C}_{\mathrm{n}}} \zeta_{\mathrm{n}}^{2}(\phi, t) \mathrm{d} \phi+\tilde{\omega}_{\mathrm{s}} \oint_{\mathscr{C}_{\mathrm{s}}} \zeta_{\mathrm{s}}^{2}(\phi, t) \mathrm{d} \phi
$$

remains equal to its initial value for all time (Dritschel 1988b). Equation (18) restricts the mean-square contour displacements since each term has the same sign. Instability therefore requires $\tilde{\omega}_{\mathrm{n}} \tilde{\omega}_{\mathrm{s}}<0$, at least.

We next pursue this part of parameter space. To cut down to the fewest essential parameters possible, we are free to choose a dimensionalization for the vorticity, and the one adopted makes the average vorticity jump into the band equal to unity:

$$
\frac{1}{2}\left(\tilde{\omega}_{\mathrm{s}}-\tilde{\omega}_{\mathrm{n}}\right)=\omega_{\mathrm{b}}-\frac{1}{2}\left(\omega_{\mathrm{n}}+\omega_{\mathrm{s}}\right)=1 .
$$

Because of the restriction of zero total circulation (2), the only free vorticity parameter is

$$
\omega_{\mathrm{ns}}=\tilde{\omega}_{\mathrm{n}}+\tilde{\omega}_{\mathrm{s}}=\omega_{\mathrm{n}}-\omega_{\mathrm{s}}
$$

by (3). $\omega_{n s}$ is the difference between the vorticity north and south of the band. The simplest Rayleigh problem has $\omega_{\mathrm{ns}}=0$. 

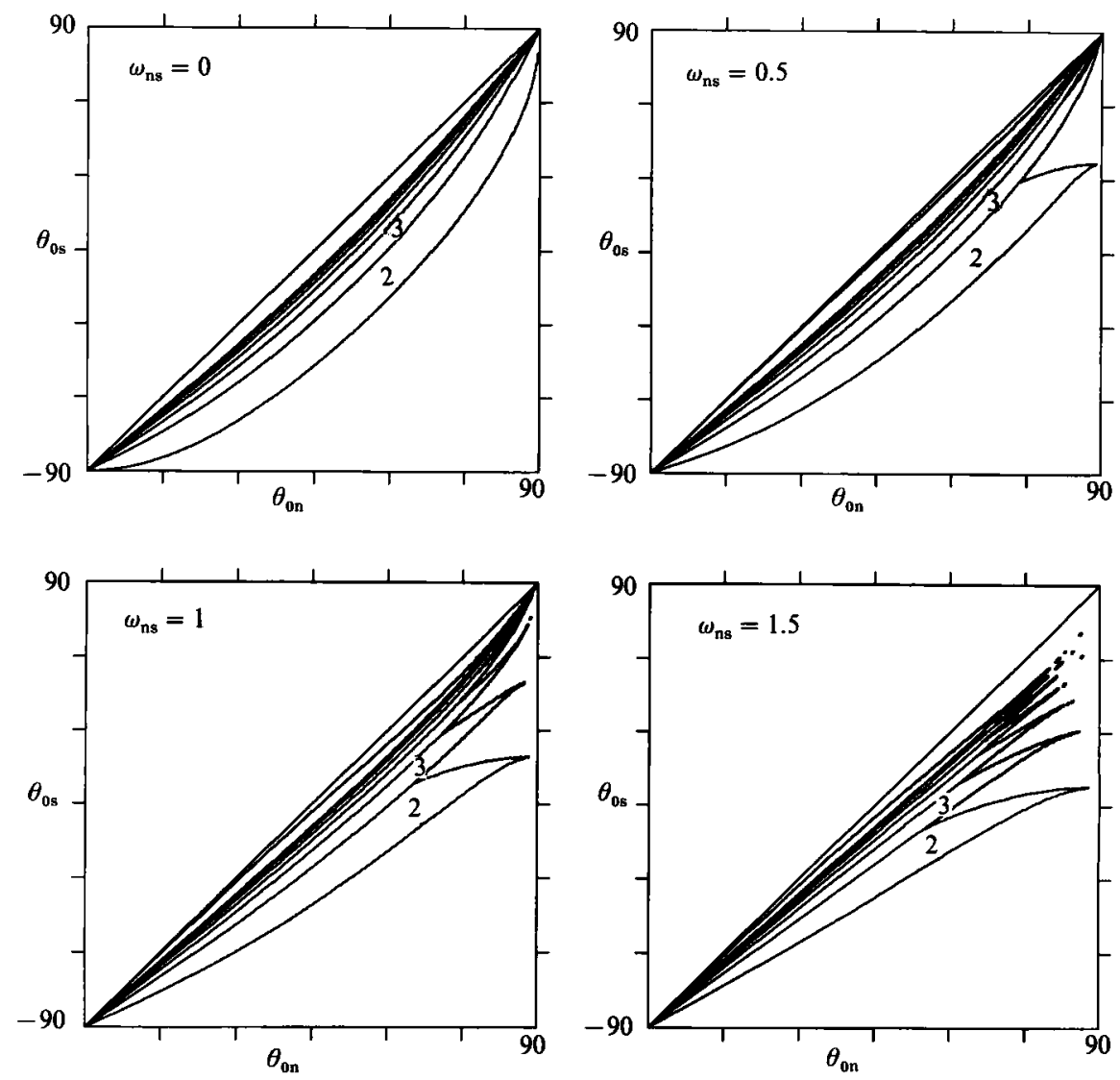

Figure 3. Contour maps of the most unstable mode $m$ corresponding to figure 2 . The first few regions are labelled for clarity.

The essential parameters are therefore $\theta_{0 \mathrm{n}}, \theta_{\mathrm{os}}$ and $\omega_{\mathrm{ns}}$ (recall $z=\sin \theta$ ), and of course the azimuthal wavenumber $m$ of the disturbance. Because $\tilde{\omega}_{\mathrm{n}} \tilde{\omega}_{\mathrm{s}}<0, \omega_{\mathrm{ns}}$ must lie between -2 and 2 . In fact, it is sufficient to consider $\omega_{n s} \geqslant 0$ because of north-south symmetry (the subscripts ' $n$ ' and ' $s$ ' can be exchanged everywhere).

Figure 2 shows contour maps of the maximum growth rate (the imaginary part of $\sigma$, denoted $\sigma_{1}$ ) as a function of $\theta_{0 \mathrm{n}}$ and $\theta_{\mathrm{os}}$, for four selected values of $\omega_{\mathrm{ns}}$. The maximum is taken over the first eight azimuthal wavenumbers. The corresponding azimuthal mode of maximum instability is contoured in figure 3 using the same format. The results for $\omega_{\mathrm{ns}}<0$ can be obtained by reflection across the diagonal connecting the upper left to the lower right corner of each map.

The first surprise is that the mode $m=2$ can be unstable, even when $\omega_{\mathrm{ns}}=0$. There is no analogue of this in the planar case : a circular band of vorticity is always stable to $m=2$ disturbances (cf. figure 2 of Dritschel 1986, and references therein). The peak growth rate for $m=2$ when $\omega_{\text {ns }}=0$ is $\frac{1}{8}(5 \sqrt{ } 5-11)^{\frac{1}{2}} \approx 0.1501$, and this occurs when $\theta_{0 \mathrm{n}}=-\theta_{0 \mathrm{~s}}=\sin ^{-1}(\sqrt{ } 5-2) \approx 13.65^{\circ}$.

The peak instability for higher wavenumbers shifts toward the line $\theta_{0 \mathrm{n}}=\theta_{0 \mathrm{~s}}$, corresponding to narrower bands. This occurs also in the planar case: the wavenumber tends to scale with the inverse thickness of the band (the thin band limit and its connection with the planar result is discussed in Appendix B). Non-zero $\omega_{n s}$ shifts the location of maximum instability to more southern latitudes (a band 
(a)
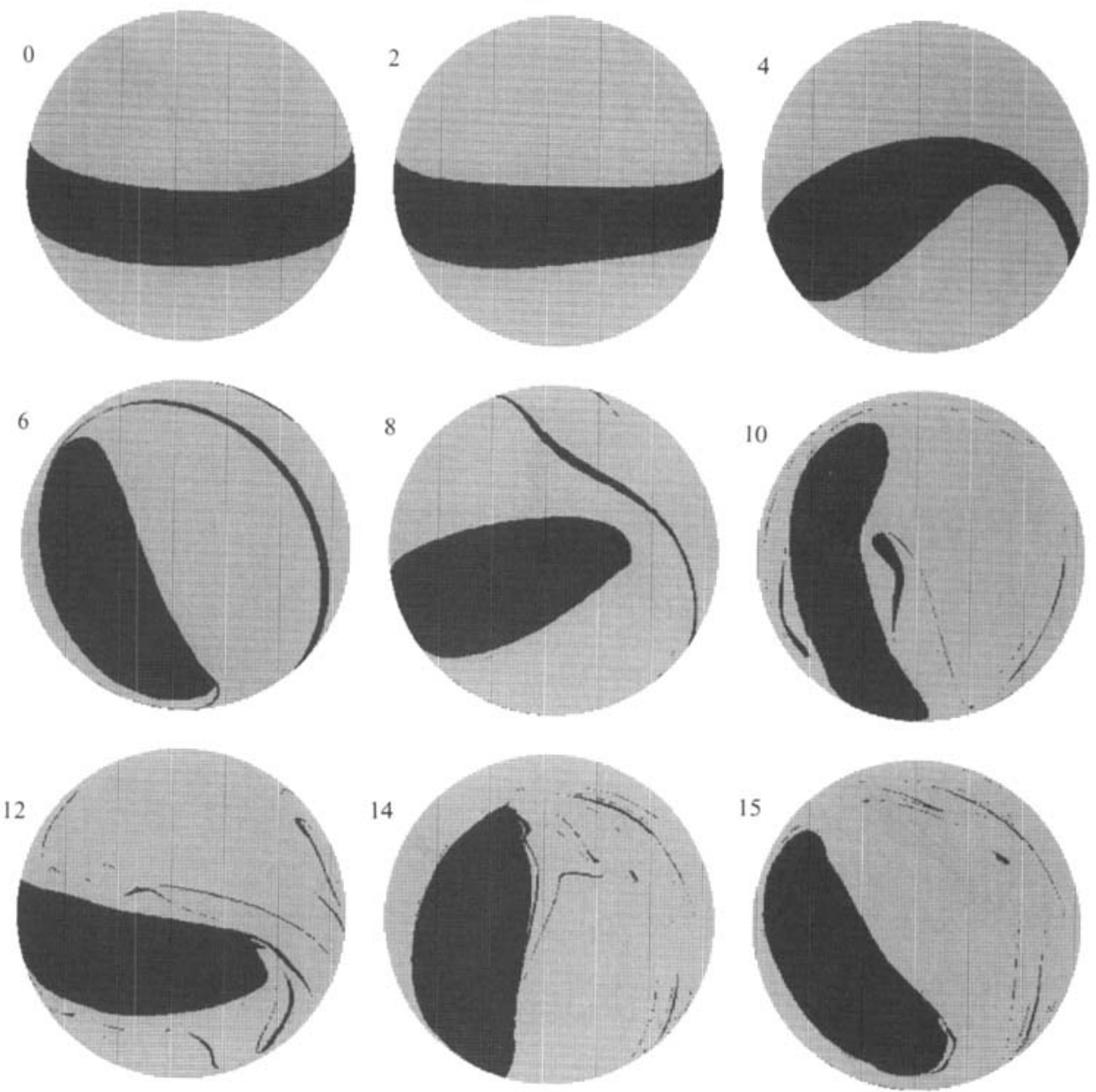

Figure $4(a)$. For caption see facing page.

located in the southern hemisphere is more unstable than one in the northern hemisphere when the vorticity north of the band exceeds that south of the band). As $\omega_{\text {ns }}$ approaches 2 , the growth rates decrease before vanishing entirely at $\omega_{\mathrm{ns}}=2$, when the distribution of vorticity becomes monotonic and therefore incapable of supporting instabilities.

We next illustrate a few of these instabilities by direct numerical simulation of (1). The numerical algorithm, 'contour surgery', is fully documented in Dritschel (1989 $b$ and references therein), where one can find details of the numerical parameters and wide-ranging tests of accuracy. Suffice it to say that the calculations presented are performed at sufficient spatio-temporal resolution to be reproducible on all but the smallest spatial scales.

In the first simulation, $\omega_{\mathrm{ns}}=0$, and the basic state is taken to be the one most unstable to the $m=2$ disturbance, $\theta_{\text {on }}=-\theta_{0 \mathrm{~s}} \approx 13.65^{\circ}$. Only the northern contour is disturbed, with $\theta_{\mathrm{n}}(\phi, 0)=\theta_{0 \mathrm{n}}+1^{\circ} \cos 2 \phi$ initially, to allow the flow to develop without imposition of north-south symmetry. The development and maturation of the resulting instability is depicted in figures $4(a)$ and $4(b)$, with $(b)$ showing a view from the opposite side of the sphere. The band rolls up into two giant vortices, each 
(b)
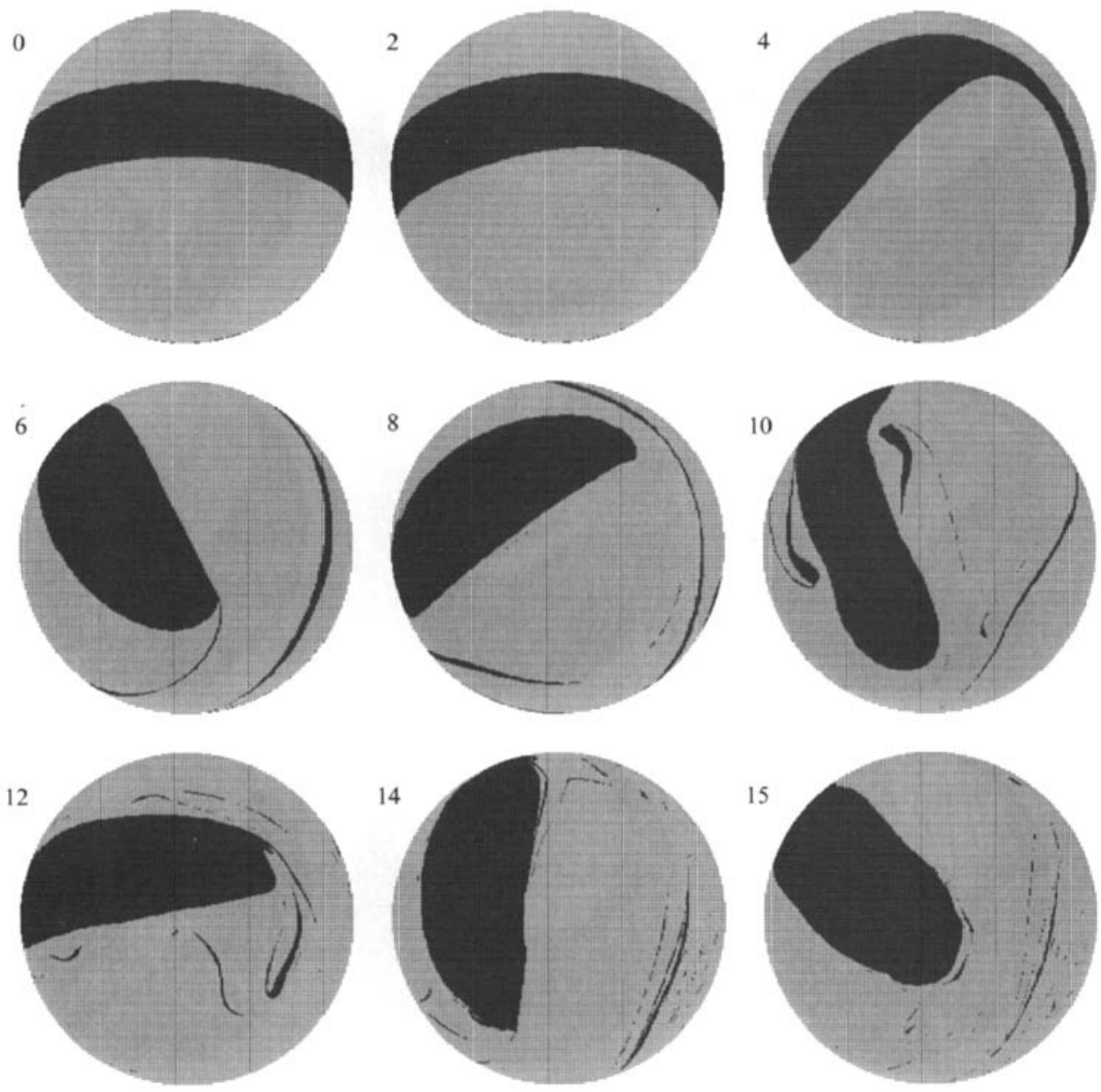

Figure 4. The nonlinear development of the unstable basic flow $\theta_{0 \mathrm{n}}=-\theta_{\mathrm{os}} \approx 13.65^{\circ}, \omega_{\mathrm{ns}}=0$. The time, $t$, given in the upper left-hand corner of each frame, is scaled by the factor $2 \pi$. The numerical parameters in this and subsequent simulations are $\mu=0.06, \delta=0.00045$, and $\Delta t=0.05$. (a) Orthographic view from $\theta=20^{\circ}, \phi=20^{\circ}$. (b) View from the opposite side of the sphere.

occupying about a quarter of the surface of the sphere. Then, some of the filamentary vorticity caught between the two vortices thickens as it is swept toward the sides of the two elongated vortices. A dimple forms along the sides of each vortex which is subsequently carried around to the vortex tips. There, the dimple is greatly exaggerated by the local straining field (see Dritschel 1988c, $1989 b$ for remarks), leading to the expulsion of thick filaments of vorticity. The vortices suffer a reduction in aspect ratio immediately afterwards which appears to stabilize the vortices against further such events. Caught between the vortices is a swarming mass of filaments largely kept at bay by the strong differential rotation induced by the two vortices. Why little roll-up is observed among these filaments will be explained in the next section.

The second simulation illustrates the effect on non-zero $\omega_{n s}$. Now, we take $\omega_{n s}=$ -1 , with $\theta_{0 \mathrm{~s}}=33^{\circ}$ and $\theta_{0 \mathrm{n}}=44^{\circ}$. This basic configuration is close to the one most unstable to $m=3$. Again, only the northern contour is disturbed, with a $1^{\circ}$ amplitude 

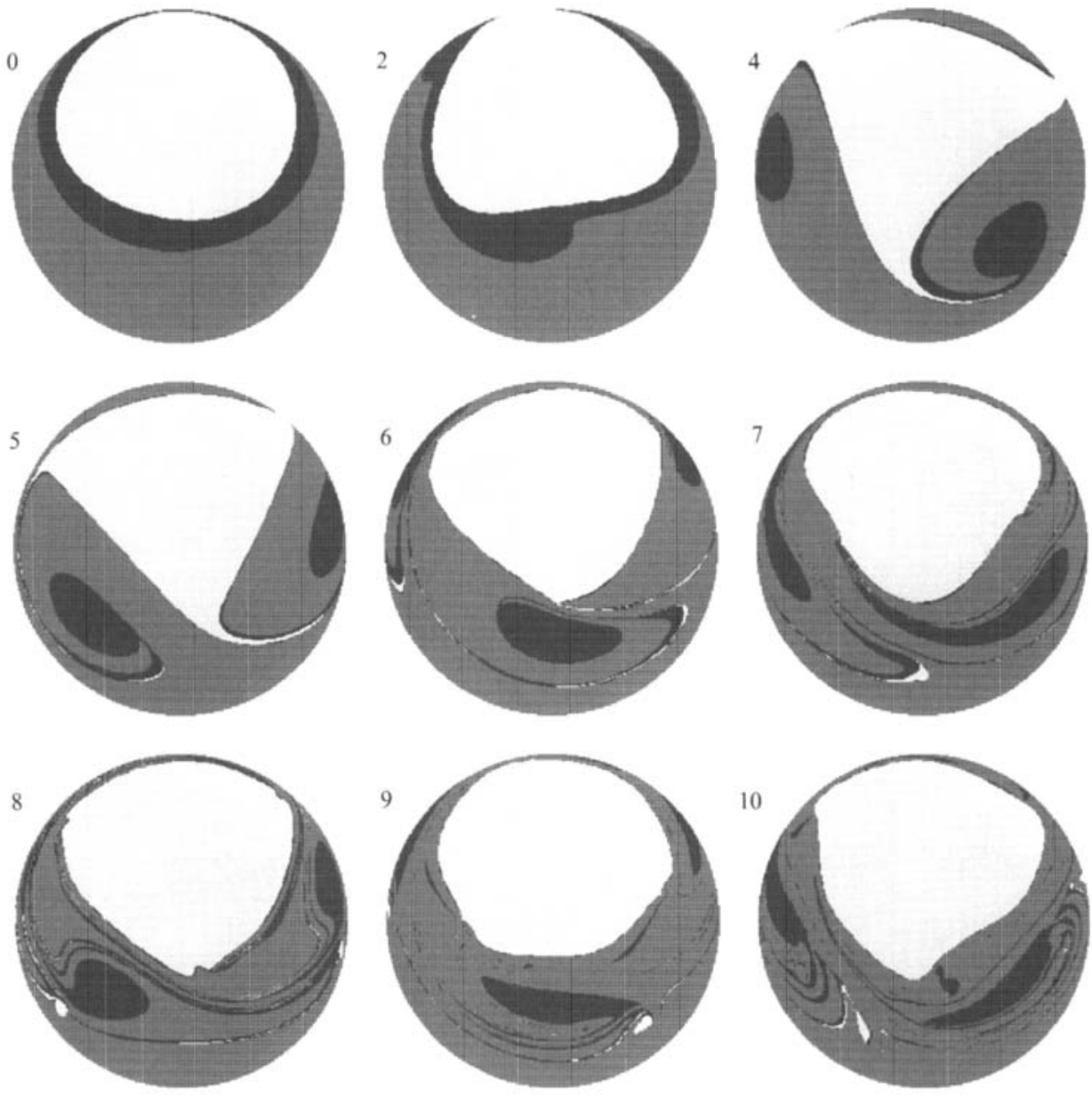

Figure $5(a)$. For caption see facing page.

$\left(\theta_{\mathrm{n}}(\phi, 0)=\theta_{\mathrm{0n}}+1^{\circ} \cos 3 \phi\right)$. Figures $5(a)$ and $5(b)$ depict the evolution from an orthographic and stereographic perspective. Again, the vortices roll-up, but before they have a chance to fully organize, they are extended by the strong differential rotation of the basic flow (the effect of non-zero $\omega_{n s}$ ). The vortices continually battle with the differential rotation to remain intact, periodically extending and consolidating depending on the phase difference between the wave on the polar contour and the vortices. Each interaction produces further filamentary debris, rapidly increasing the complexity of the flow.

Calculations performed with higher azimuthal wavenumber and thinner bands show less sensitivity to the curvature of the sphere's surface. Away from the polar regions, a thin band appears locally straight and flat, and the instabilities develop accordingly (see also Appendix B). Likewise, near one of the poles, the band lies in an approximately horizontal surface, and then the band behaves like an annular vortex in planar geometry (some examples of this evolution may be found in Dritschel 1986). 
(b)
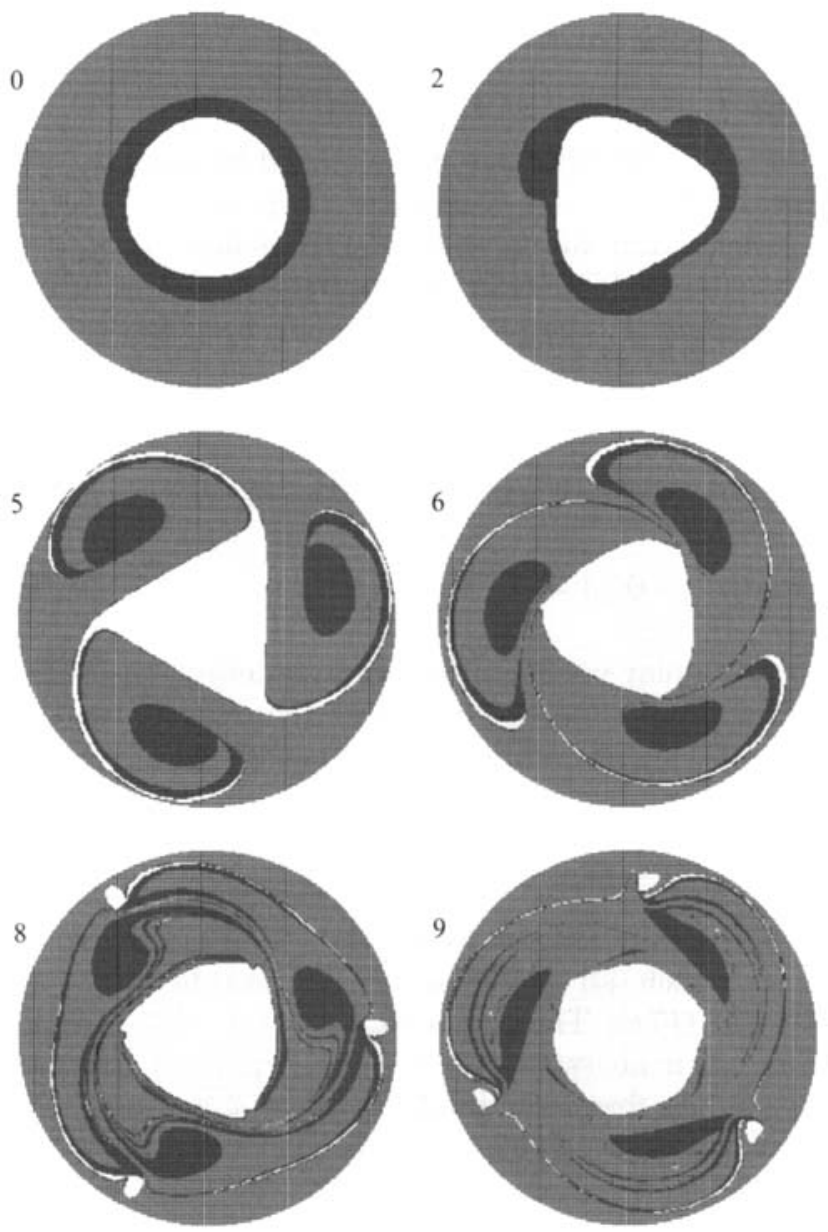
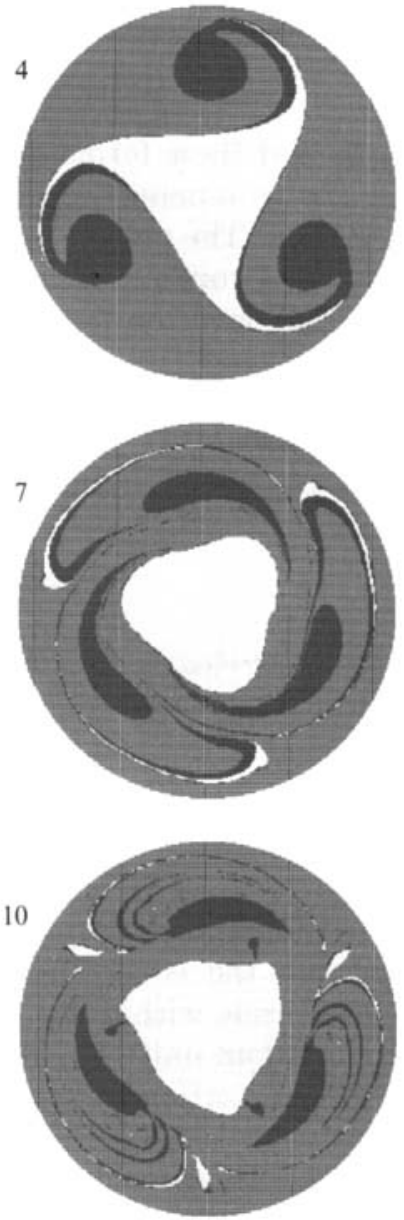

FigURe 5. The nonlinear development of the unstable basic flow $\theta_{0 \mathrm{n}}=44^{\circ}, \theta_{0 \mathrm{~s}}=33^{\circ}$, with $\omega_{\mathrm{ns}}=-1$. (a) View from $\theta=60^{\circ}, \phi=20^{\circ}$. (b) Polar stereographic view looking down on the north pole (the equator is at the edge of the disc).

\section{Stability in the presence of polar vortices}

We next examine the effect of the presence of two polar vortices on the preceding stability results. The motivation comes from the recent numerical experiments of Juckes \& McIntyre (1987), who have observed the roll-up of vorticity filaments in the vicinity of a polar vortex. This roll-up is surprising in view of the strong adverse shear produced by the vortex which should make the filaments stable.

For generality, we consider then a vortex of strength $\kappa_{\mathrm{np}}(=$ circulation $/ 2 \pi)$ placed at the north pole, and another of strength $\kappa_{\text {sp }}$ is placed at the south pole. Each vortex, for maximum simplicity, is assumed to be concentrated at a point, though we have in mind applying the results to finite polar vortices. This is justified as long as the equivalent finite vortices remain relatively undisturbed by the action of the band between them. Some justification comes from analogous planar results, for which a direct comparison between point and finite vortices has been made (Dritschel 1989a), 
while more direct justification is given below in a nonlinear calculation that uses finite vortices.

The stream functions associated with the point vortices are

$$
\psi_{\mathrm{np}}(z)=\frac{1}{2} \kappa_{\mathrm{np}} \log (1-z), \quad \psi_{\mathrm{sp}}(z)=\frac{1}{2} \kappa_{\mathrm{sp}} \log (1+z) .
$$

Recall that these forms take into account both the flow created by the point vortex and the compensating uniform background vorticity that ensures zero total circulation. The principle of superposition allows us to add these flows to that due to the band of vorticity already considered. If we restrict attention to disturbances with azimuthal wavenumbers greater than 1 , then the two polar vortices will not be disturbed, and the stability analysis is then scarcely altered from before. We just need to augment $\Omega_{0 \mathrm{n}}$ and $\Omega_{0 \mathrm{~s}}$, the angular velocities at the edge of band, by the point vortex flows (21).

The additional angular velocity associated with the polar vortices is given by

$$
\Omega_{\mathrm{p}}(z)=-\frac{\partial}{\partial z}\left(\psi_{\mathrm{np}}+\psi_{\mathrm{sp}}\right)=\frac{\kappa_{\mathrm{np}}}{2(1-z)}-\frac{\kappa_{\mathrm{sp}}}{2(1+z)} .
$$

To see most clearly the effect of the polar vortices, the original definitions of $\Omega_{0 n}$ and $\Omega_{0 \text { s }}$ will be left unchanged in lieu of altering the final stability results in (17). The definition of $\sigma$ is the same as written in $(17 a)$, while $(17 b)$ and $(17 c)$ acquire additional terms :

$$
\begin{aligned}
& \omega_{+} \equiv \frac{1}{2}\left(\tilde{\omega}_{\mathrm{n}}+\tilde{\omega}_{\mathrm{s}}\right)-m\left(\Omega_{0 \mathrm{n}}+\Omega_{\mathrm{os}}\right)-m\left(\Omega_{\mathrm{p}}\left(z_{0 \mathrm{n}}\right)+\Omega_{\mathrm{p}}\left(z_{0 \mathrm{~s}}\right)\right) \\
& \omega_{-} \equiv \frac{1}{2}\left(\tilde{\omega}_{\mathrm{n}}-\tilde{\omega}_{\mathrm{s}}\right)-m\left(\Omega_{0 \mathrm{n}}-\Omega_{0 \mathrm{~s}}\right)-m\left(\Omega_{\mathrm{p}}\left(z_{0 \mathrm{n}}\right)-\Omega_{\mathrm{p}}\left(z_{0 \mathrm{~s}}\right)\right) .
\end{aligned}
$$

To understand the potentially stabilizing role of the polar vortices, let us determine the conditions under which all disturbances are stable. Consider the various terms within the radical in $(17 a)$. The terms $\left|\tilde{\omega}_{\mathrm{n}} \tilde{\omega}_{\mathrm{s}}\right|\left(=\left|1-\frac{1}{4} \omega_{\mathrm{ns}}^{2}\right|\right)$ and $S$ are both less than unity (recall $\tilde{\omega}_{\mathrm{n}} \tilde{\omega}_{\mathrm{s}}<0$ is necessary for instability). With the vorticity dimensionalization adopted in (19), the first term in $(23 b), \frac{1}{2}\left(\tilde{\omega}_{\mathrm{n}}-\tilde{\omega}_{\mathrm{s}}\right)$, equals -1 . The second term, $-m\left(\Omega_{0 \mathrm{n}}-\Omega_{0 \mathrm{~s}}\right)$, is always positive as long as $\left|\omega_{\mathrm{ns}}\right|<2$, and this latter condition is satisfied as a result of $\tilde{\omega}_{n} \tilde{\omega}_{s}<0$. Hence, if the third term, $-m\left(\Omega_{\mathrm{p}}\left(z_{0 \mathrm{n}}\right)-\Omega_{\mathrm{p}}\left(z_{0 \mathrm{~s}}\right)\right)$ is sufficiently negative to overcome the second one, then $\left|\omega_{-}\right|$ will be greater than 1 . This is enough to ensure that the quantity within the radical will be positive for all disturbance wavenumbers $m$. Therefore, a sufficient condition for stability is that the total angular velocity difference across the band be of opposite sign to the angular velocity difference due to the band alone. To put it mathematically,

$$
A \equiv-\frac{\Omega_{\mathrm{p}}\left(z_{0 \mathrm{n}}\right)-\Omega_{\mathrm{p}}\left(z_{0 \mathrm{~s}}\right)}{\Omega_{\mathrm{0n}}-\Omega_{\mathrm{os}}} \geqslant 1
$$

is a second sufficient condition for stability. There is stability either when $\tilde{\omega}_{\mathrm{n}} \tilde{\omega}_{\mathrm{s}} \geqslant 0$ or when $A \geqslant 1$. This second condition corresponds to stabilization by adverse shear (Dritschel 1989a). In both the spherical and planar cases, a reversal in the angular or linear velocities brings about stability. (The reason why there should be a close link between these cases is discussed in Dritschel 1988b.)

What is different about spherical flows is the nature of the adverse shear produced by the polar vortices. To illustrate this without being encumbered by the great number of parameters already present, several simplifications are made. First, the band is taken to be very thin, i.e. $\left(z_{0 \mathrm{n}}-z_{0 \mathrm{~s}}\right) \ll\left(z_{0 \mathrm{n}}+z_{0 \mathrm{~s}}\right)$. In this case, we may introduce the dimensionless parameter $k \equiv m\left(\theta_{0 \mathrm{n}}-\theta_{0 \mathrm{~s}}\right)$ in place of $m$ and refer only to the mean latitude of the band, $\bar{\theta}_{0} \equiv \frac{1}{2}\left(\theta_{0 \mathrm{n}}+\theta_{0 \mathrm{~s}}\right)\left(\bar{z}_{0}=\sin \bar{\theta}_{0}\right.$ will be used also $)$. 
Second, the vorticity on either side of the band is taken to be equal, so $\omega_{\mathrm{ns}}=0$. Lastly, we confine our attention to the three simplest polar flows: $(a) \kappa_{\mathrm{np}}=\kappa_{\mathrm{sp}}=\kappa$, (b) $\kappa_{\mathrm{np}}=\kappa, \kappa_{\mathrm{sp}}=0$ and $(c) \kappa_{\mathrm{np}}=-\kappa_{\mathrm{sp}}=\kappa$. Case $(a)$ for two equal polar vortices is relevant to the calculation in figure 4 , case $(c)$ for two opposite polar vortices is relevant to the hemispherical simulations of Juckes \& McIntyre (1987), and case (b) corresponds to the more realistic terrestrial situation where a single polar vortex is present.

With these simplifications, the band is locally flat and straight, so we can apply the known planar results (see Dritschel $1989 a$ ). It is only necessary to calculate the adverse shear $\Lambda$ for the three polar flows considered. For case $(a)\left(\kappa_{\mathrm{np}}=\kappa_{\mathrm{sp}}=\kappa\right),(24)$ gives

$$
A=\frac{\kappa}{\tilde{\omega}_{\mathrm{b}}}\left(\frac{1+\bar{z}_{0}^{2}}{1-\bar{z}_{0}^{2}}\right),
$$

where we have explicitly shown the dependence on the vorticity jump into the band $\tilde{\omega}_{\mathrm{b}}$, previously fixed at unity by (19). $\tilde{\omega}_{\mathrm{b}}$ and $\kappa$ will both be considered positive in the following discussion. For case $(b)\left(\kappa_{\mathrm{np}}=\kappa, \kappa_{\mathrm{sp}}=0\right)$,

$$
\Lambda=\frac{\kappa}{2 \tilde{\omega}_{\mathrm{b}}}\left(\frac{1+\bar{z}_{0}}{1-\bar{z}_{0}}\right),
$$

and for case $(c)\left(\kappa_{\mathrm{np}}=-\kappa_{\mathrm{sp}}=\kappa\right)$,

$$
\Lambda=\frac{2 \kappa}{\tilde{\omega}_{\mathrm{b}}}\left(\frac{\bar{z}_{0}}{1-\bar{z}_{0}^{2}}\right) .
$$

For $\bar{z}_{0}$ close to 1 , all three expressions reduce to the planar result,

$$
\Lambda=\frac{2 \kappa}{\tilde{\omega}_{\mathrm{b}}}\left(\frac{1}{\vec{r}_{0}^{2}}\right),
$$

where $\bar{r}_{0} \equiv\left(1-\bar{z}_{0}^{2}\right)^{\frac{1}{2}}$ is the mean distance of the band from the polar axis (Dritschel $1989 a$ ). Away from the polar regions, however, all three expressions imply considerably less adverse shear than one would estimate, say, using cord distance $\left[2\left(1-\bar{z}_{0}\right)\right]^{\frac{1}{2}}$ in place of $\bar{r}_{0}$ in (26). Indeed, $\Lambda$ vanishes at the south pole in case $(b)$, and in case $(c)$ it changes sign at the equator and takes on negative values in the southern hemisphere (which implies an even greater tendency for instability). Case (c) is the most relevant to the work of Juckes \& McIntyre (1987) since the circulation in the two hemispheres is equal in magnitude but opposite in sign.

Setting $\Lambda=1$ in $(25 a-c)$, we can determine the region of the spherical surface in which a thin band of vorticity will be stabilized by adverse shear. This will depend on the ratio of the polar vortex strength $\kappa$ to the vorticity jump into the band $\tilde{\omega}_{\mathrm{b}}$. This result is shown in figure 6 for the three cases. More relevant to the nonlinear problem of vortex roll-up, however, is the same figure for $\Lambda=\Lambda_{\mathrm{c}}=0.65$, the value of the adverse shear below which bands of vorticity exhibit irreversible disruption (Dritschel $1989 a$ ). The result in this case can be obtained by using $2 \kappa / \tilde{\omega}_{\mathrm{b}} \boldsymbol{A}_{\mathrm{c}}$ in place of $2 \kappa / \tilde{\omega}_{\mathrm{b}}$ in figure 6 .

As expected, when the polar vortices are weak they cannot prevent instability. What is significant however, is that, in cases $(b)$ and especially $(c)$, a substantial area of the spherical surface is ripe for the roll-up of filaments even for large values of $2 \kappa / \tilde{\omega}_{\mathrm{b}}$. Essentially, thin filaments of vorticity in the equatorial regions of the northern hemisphere and over the whole southern hemisphere are unstable to roll-up, even when the polar vortex is several times stronger than the filament. When the 


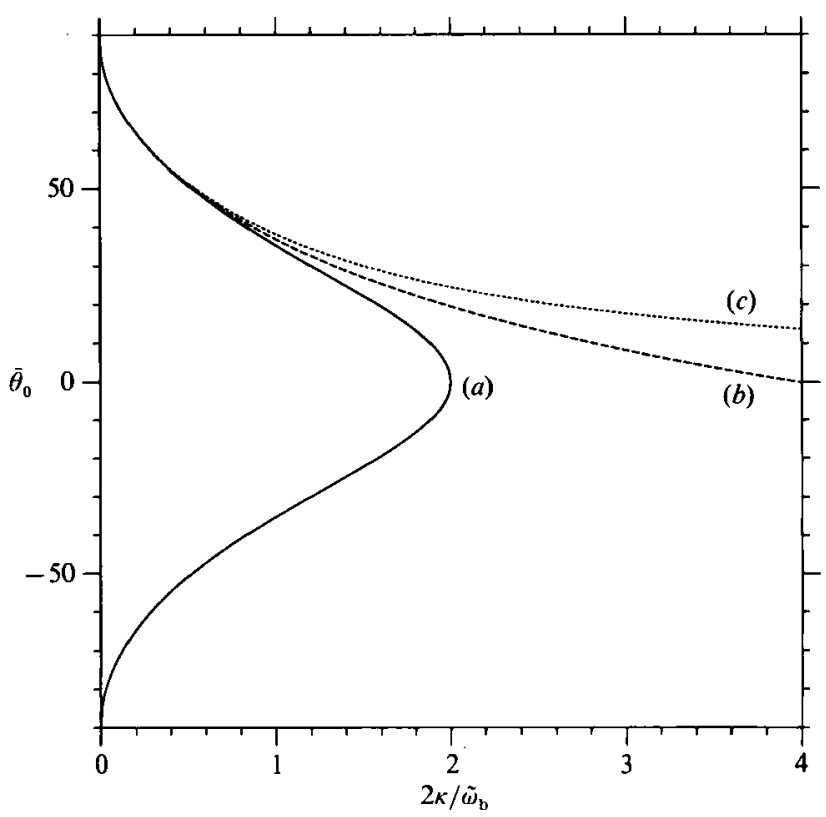

Figure 6. Stability regions as a function of $2 \kappa / \tilde{\omega}_{\mathrm{b}}$ for the three polar vortex configurations. The case of equal vortices is given by the solid curve $(a)$; the regions outside this curve are stable. The case of one polar vortex is shown by the dashed curve $(b)$; the stable region lies north of it. And, the case of two opposite polar vortices is shown by the dotted curve $(c)$; the stable region again lies north of it.

strengths are comparable, figure 6 shows that any filament south of approximately $40^{\circ}$ latitude will be unstable.

Case $(a)$, which exhibits much greater stability, is relevant to the calculation shown in figure 4. It explains why the filaments caught between the two large, likesigned vortices are able to resist roll-up at late times.

We conclude this section with a nonlinear calculation that demonstrates the general inability of antisymmetrically distributed polar vortices to prevent roll-up. Finite polar vortices are used in the calculation illustrated. The northern vortex extends down to a latitude of $50^{\circ}$, and the vorticity jump across its edge is $\tilde{\omega}_{\mathrm{np}}=1$. The southern vortex extends up to $-40^{\circ}$, and has the same vorticity jump ( $\kappa_{\mathrm{sp}}=$ $-\left(1-\sin 40^{\circ}\right)$ while $\left.\kappa_{\mathrm{np}}=1-\sin 50^{\circ}\right)$. The slight imbalance in vortex strengths is approximately compensated for by a band of finite width straddling the latitudes between $10^{\circ}$ and $20^{\circ}$ north. The vorticity jump into the band is $\tilde{\omega}_{\mathrm{b}}=\frac{1}{2}$, or half of that at the edge of each polar vortex. Numerical noise provides the initial disturbance.

Figures $7(a)$ and $7(b)$ show the evolution using orthographic and polar stereographic projections. The band rapidly destabilizes and disrupts the northern polar vortex (see figure $7 c$ for an enlargement of $7 b$ at the latest time). The band causes this disruption by developing asymmetrically, with pronounced lowwavenumber components. These low-wavenumber components have a long range of interaction and so disturbances are readily transmitted between the band and the polar vortex.

A little more insight can be gained by calculating the adverse shear initially experienced by the band. The relevant formulae are (22) and (24). When one substitutes the numerical parameters characterizing the initial flow, one obtains the value $\Lambda=0.208$, which falls substantially short of the value for stabilization $(\Lambda=$ 
(a)
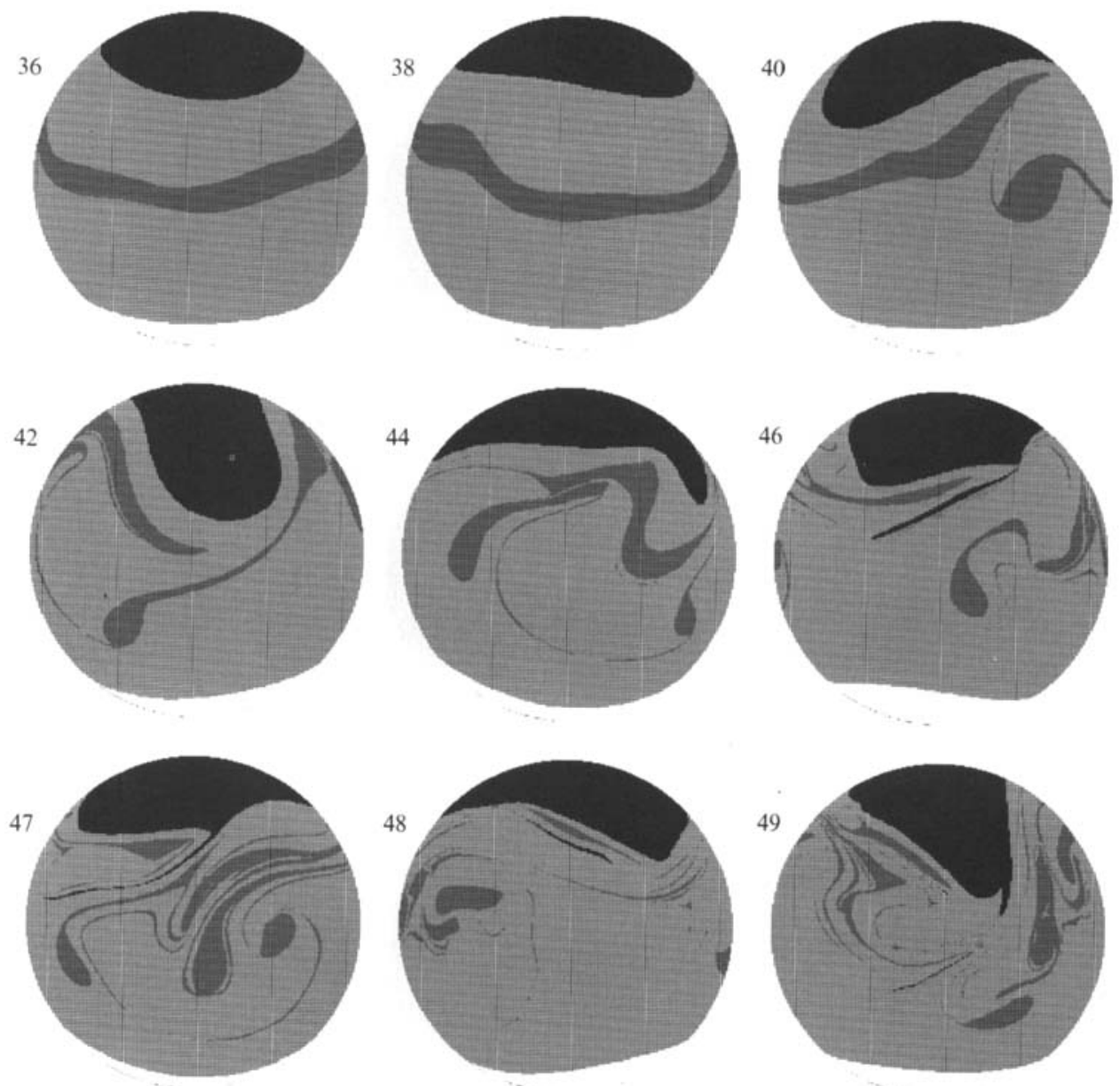

Fiqure $7(a)$. For caption see p. 63.

1). Furthermore, this value of adverse shear lies on the boundary between two nonlinear regimes in the evolution of planar bands, as discussed in Dritschel $(\mathbf{1 9 8 9} a)$. For $\Lambda \lesssim 0.21$, the band rolls up immediately into a string of vortices, while for $0.21 \lesssim \Lambda \lesssim 0.45$, the initial roll-up does not complete before the shear extends the enlarged sections of the band, creating a complex sequence of folds and miniature roll-ups. Both types of behaviour are evident in figure 7 .

\section{A simple mechanism for band instability}

We present here a simple physical explanation for the instability of bands of vorticity, whether they be on planar or spherical surfaces. This explanation only makes use of the dispersion relation for a single interface or vorticity discontinuity.

In a rotating or translating frame of reference, depending on the geometry, the frequency of any disturbance is independent of wavenumber, and takes the value $\frac{1}{2} \tilde{\omega}$, where $\tilde{\omega}$ is the vorticity jump across the interface (Dritschel $1988 c$ ). The angular or 
(b)
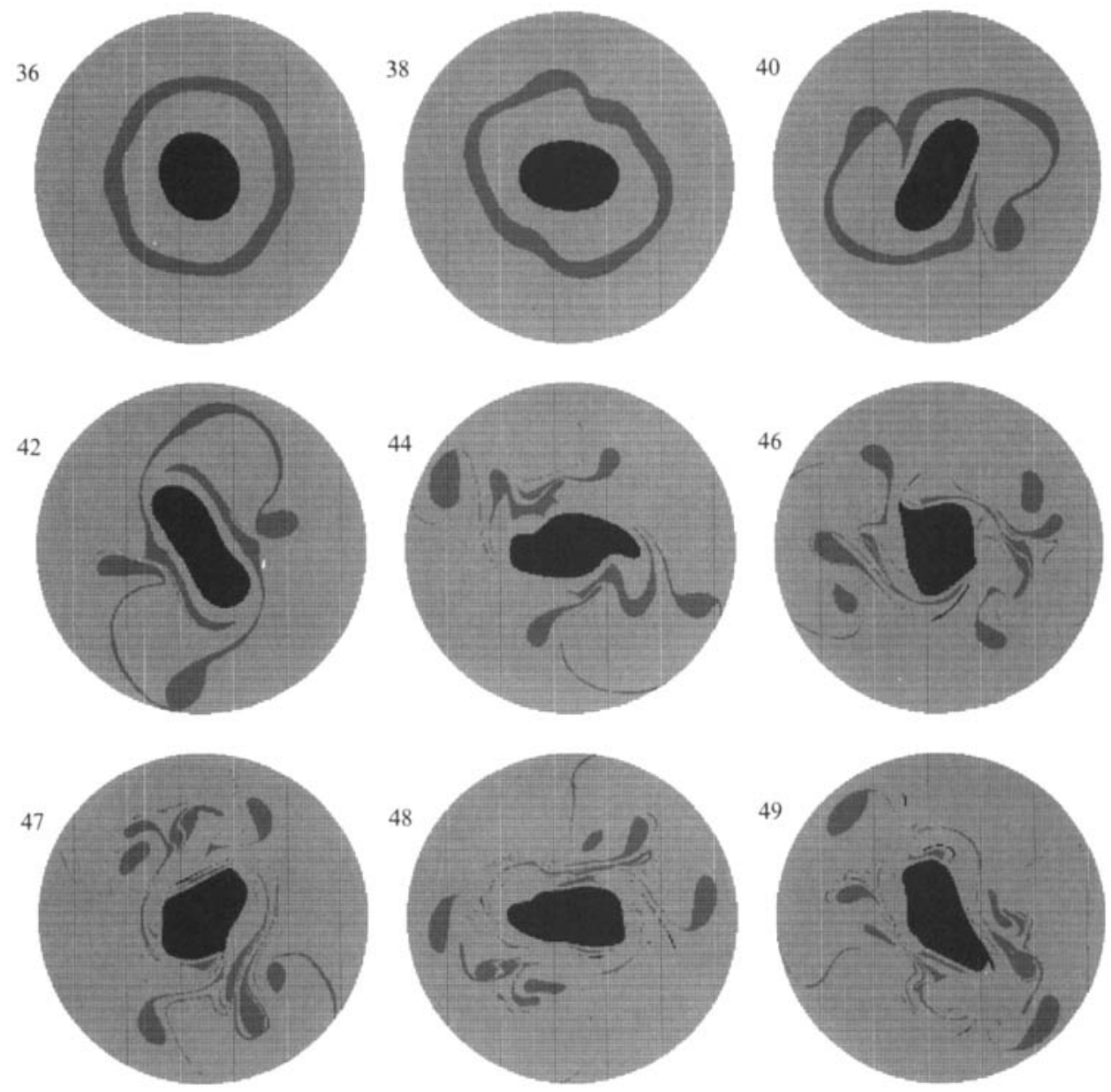

Figure $7(b)$. For caption see facing page.

linear velocity with which waves propagate is simply $-\tilde{\omega} / 2 m$, where $m$ stands for either azimuthal wavenumber or simply wavenumber for a straight, planar interface.

For a band of vorticity, for simplicity one having equal magnitude but oppositely signed vorticity jumps at its two edges, the waves on the two interfaces travel in opposite directions, omitting the basic flow contribution. This latter contribution consists of two parts: the first is the contribution from the band, which always opposes the intrinsic relative wave motion, and the second is the contribution from external regions, such as the polar vortices considered in the previous section (this contribution may either oppose or augment the intrinsic relative wave motion).

A simple yet remarkably accurate determination of the most unstable disturbance is to find, if possible, the disturbance wavenumber $m$ for which the waves on the two interfaces move together. This is called 'phase-locking' and is always possible when the flow is unstable. A little further thought about the solutions of Laplace's equation also provides an accurate estimate of the corresponding growth rate.

For simplicity, consider an annular band of vorticity in planar geometry. The 


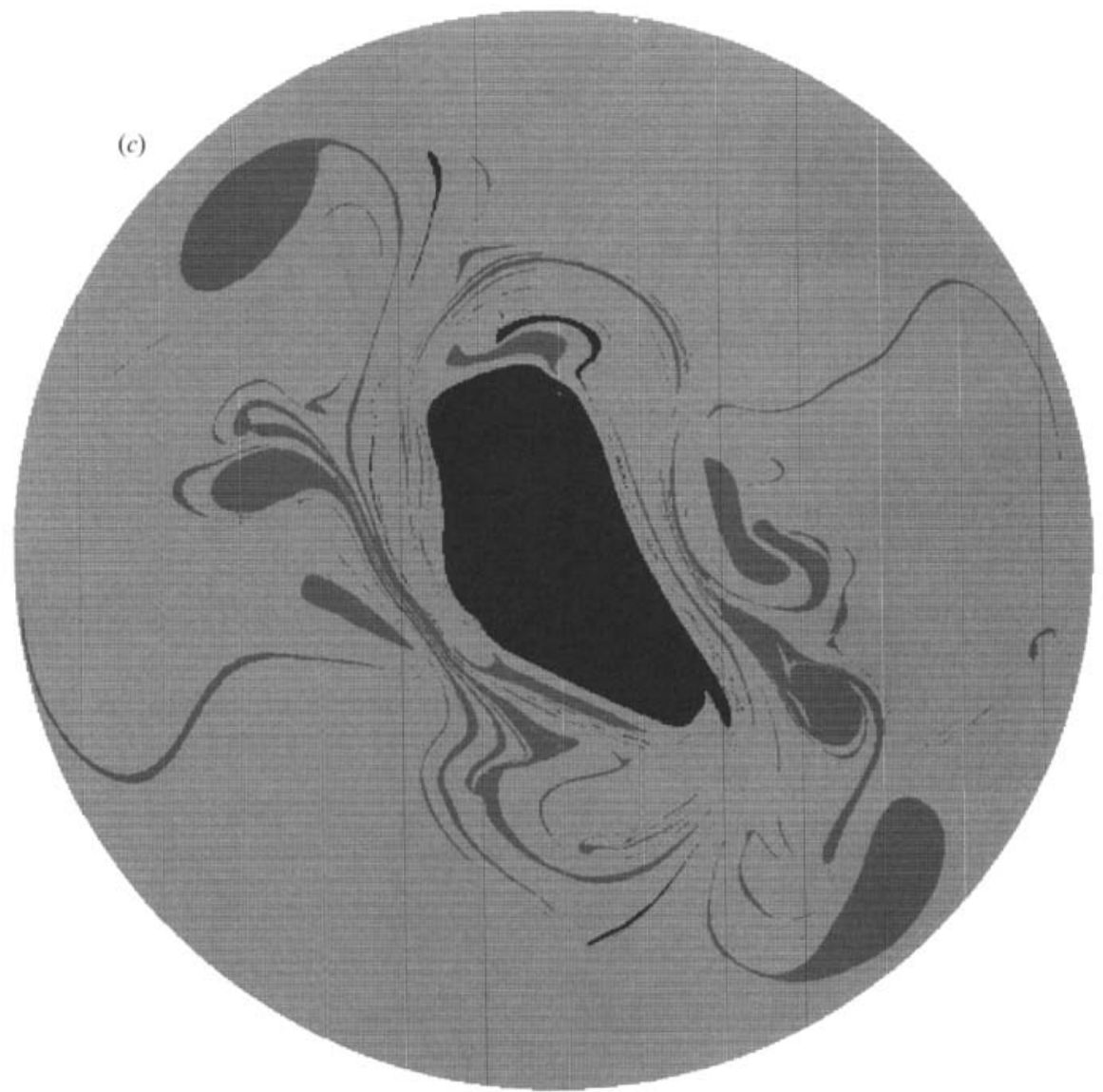

Figure 7. The nonlinear development of an unstable band lying between $\theta_{0 \mathrm{~s}}=10^{\circ}$ and $\theta_{0 \mathrm{n}}=20^{\circ}$, in the presence of two finite polar vortices of oppositely signed circulation $\left(\theta_{\mathrm{np}}=50^{\circ}\right.$ and $\theta_{\mathrm{sp}}=$ $\left.-40^{\circ}\right)$. The vorticity jump into the band $\tilde{\omega}_{\mathrm{b}}=\frac{1}{2}$ is half of that across the edges of the polar vortices. (a) Orthographic view from $\theta=20^{\circ}, \phi=20^{\circ}$. (b) Polar stereographic view (the outer dise is at $\theta=$ $\left.-20^{\circ}\right)$. (c) Enlargement of the view in $(b)$ at $t=49$.

inner edge, in equilibrium, lies at $r=a$, and the outer lies at $r=b$. The vorticity jumps by $\tilde{\omega}$ crossing into the band from the exterior irrotational fluid, and by $-\tilde{\omega}$ crossing out of the band into the interior irrotational fluid. Thus, the waves propagate at the angular velocities $\Omega_{\mathrm{w}}=-\tilde{\omega} / 2 \mathrm{~m}$ and $\tilde{\omega} / 2 \mathrm{~m}$ on the outer and inner edges of the band, omitting the basic flow contribution.

The basic flow $\Omega_{\mathrm{b}}(r)$ is calculated easily from $r^{-1} \mathrm{~d}\left(r^{2} \Omega_{\mathrm{b}}\right) / \mathrm{d} r=\omega$, where $\omega$ is the band distribution of vorticity. Along the inner edge, $\Omega_{\mathrm{b}}=0$, and along the outer edge, $\Omega_{\mathrm{b}}=\frac{1}{2} \tilde{\omega}\left(1-a^{2} / b^{2}\right)$. For generality, an external flow in the form of a central point vortex is included as well. Its basic flow is $\Omega_{\mathrm{pv}}=\kappa / r^{2}$; so, along the inner edge, $\Omega_{\mathrm{pv}}=\kappa / a^{2}$, while along the outer edge, $\Omega_{\mathrm{pv}}=\kappa / b^{2}$.

To find the most unstable wavenumber, it remains to equate the total angular velocity $\Omega_{\mathrm{w}}+\Omega_{\mathrm{b}}+\Omega_{\mathrm{pv}}$ of the waves on the two edges. This gives

$$
\frac{\tilde{\omega}}{2 m}+\frac{\kappa}{a^{2}}=-\frac{\tilde{\omega}}{2 m}+\frac{\tilde{\omega}}{2}\left(1-\frac{a^{2}}{b^{2}}\right)+\frac{\kappa}{b^{2}},
$$



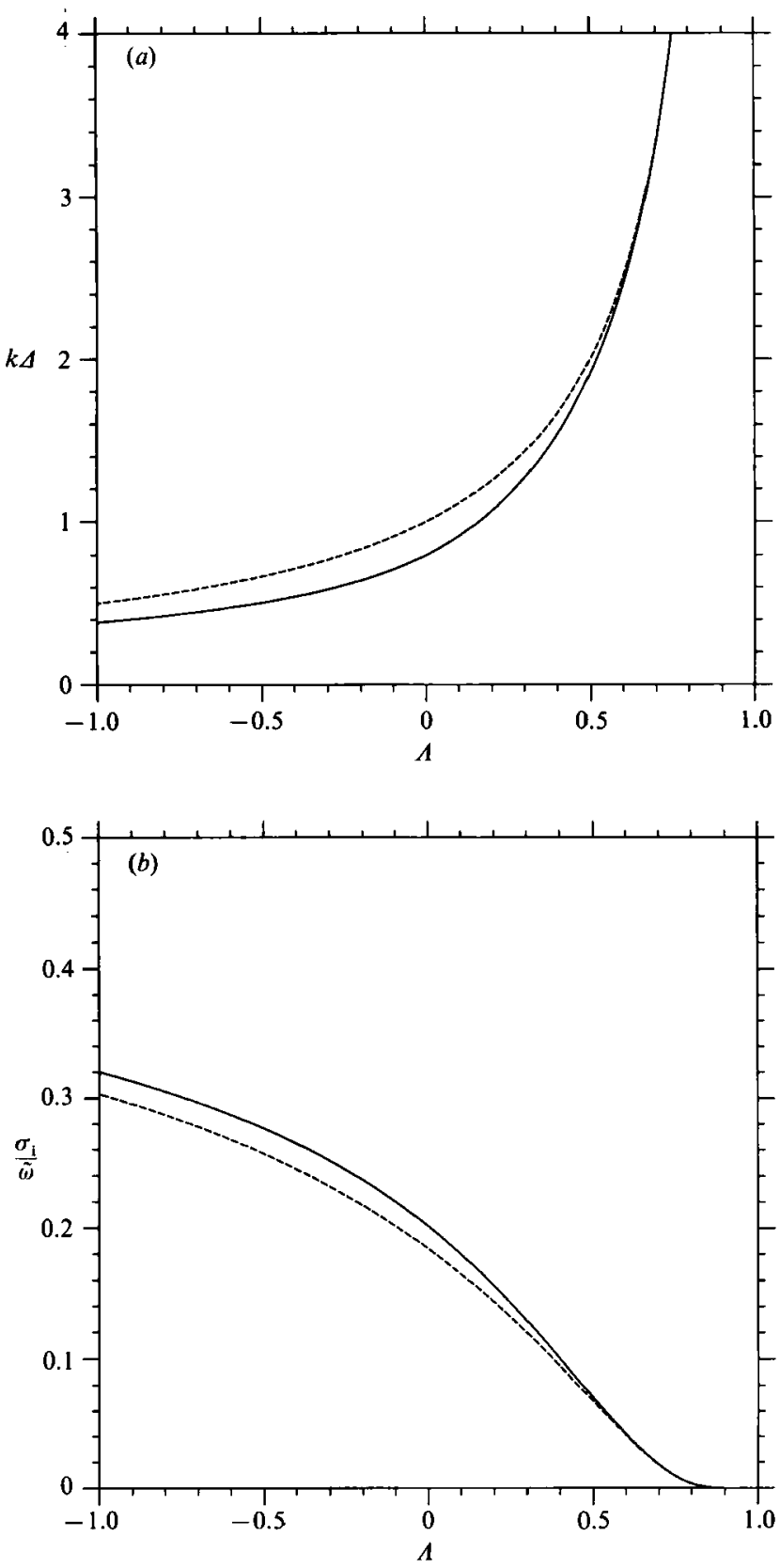

FIGURE 8. Comparison between the exact and approximate results for the linear stability of a thin band of vorticity in adverse shear $A$. (a) The dimensionless wavenumber $k \Delta$ of the most unstable mode as a function of $A$. The solid curve is the exact result obtained by a full eigenvalue analysis (Dritschel $1989 a$ ), and the dashed curve is the approximate result derived by heuristic argument. (b) The dimensionless growth rate $\sigma_{1} / \tilde{\omega}$ corresponding to $(a)$. 
which must be solved for $m$. The solution is

$$
m=\left[\frac{1}{2}\left(1-\frac{a^{2}}{b^{2}}\right)(1-\Lambda)\right]^{-1},
$$

where $A=2 \kappa / \tilde{\omega} a^{2}$ is the dimensionless shear. $\Lambda=1$ implies that the two edges of the strip move together, $\Omega_{\mathrm{b}}(a)+\Omega_{\mathrm{pv}}(a)=\Omega_{\mathrm{b}}(b)+\Omega_{\mathrm{pv}}(b)$, and (28) implies that only infinitely short waves can phase-lock. By Laplace's equation, the interaction between the waves on the two edges is then essentially zero; a sinuous streamfunction disturbance along the band implies, by irrotationality, an evanescent disturbance across the band. The higher the disturbance wavenumber $m$, the weaker the interaction between the waves on the two edges, and this accounts for the stabilization that occurs when $\Lambda$ reaches 1 . A good estimate of the growth rate of disturbances is given by the intrinsic frequency of the waves, $\frac{1}{2} \tilde{\omega}$, multiplied by the evanescent factor associated with Laplace's equation in cylindrical coordinates (the irrotational solutions of Laplace's equation are proportional to $r^{ \pm m} \mathrm{e}^{\mathrm{i} m \phi}$ ):

$$
\sigma_{1}=\frac{1}{2} \tilde{\omega}\left(\frac{a}{b}\right)^{m} .
$$

The surprising result is that (28) and (29) are good estimates even when the azimuthal wavenumber is $O(1)$. For example, when $A=0$ and $a / b=0.6$, a full linear analysis shows that $m=3$ is the only unstable eigenmode, and $\sigma_{1}=0.106 \ldots \tilde{\omega}$ (see e.g. Dritschel 1986, figure 2). For comparison, (28) predicts $m=3.125$, whose nearest integer value is 3 , while (29) predicts $\sigma_{i}=0.108 \tilde{\omega}$. Similarly close results can be obtained in the thin-strip limit, $(b-a) / a \ll 1$. Defining $\Delta \equiv(b-a)$ and $k \equiv m / a,(28)$ becomes

while (29) becomes

$$
\begin{gathered}
k \Delta=(1-\Lambda)^{-1}, \\
\sigma_{i}=\frac{1}{2} \tilde{\omega} \mathrm{e}^{-k \Delta},
\end{gathered}
$$

and both of these expressions fit well the results of the full eigenvalue analysis in this case (Dritschel $1989 a$ ) over the entire range of unstable shear values, $-\infty<A<1$ - see figures $8(a)$ and $8(b)$. The conclusion is that basic notions of wave propagation and interaction give a remarkably accurate estimate of band instability.

\section{Summary}

We have extended the classical analysis of Rayleigh to spherical geometry and have found unexpected effects. Bands of vorticity located in equatorial regions are generally more prone to instability than bands located in polar regions. A difference in the vorticity immediately north and south of the band shifts the location of maximum instability poleward; bands with a positive average inward vorticity jump are more unstable in the hemisphere of lower vorticity, and vice versa. And, thin bands of vorticity tend to be more unstable than thick bands, as in planar geometry. Overall, bands or filaments of vorticity have a more pronounced tendency to roll-up on the sphere than on the plane. Polar vortices which had been thought to be a stabilizing influence on filamentary vorticity have been shown to be largely ineffective in preventing roll-up instability, particularly in equatorial regions.

These findings may help explain the roll-up of equatorially located filaments observed in single-layer models of the Earth's stratosphere (Juckes 1987 ; Juckes \& 
McIntyre 1987; Waugh 1991), as well as the roll-up of tongues of vorticity ejected from the main polar vortex during wave breaking events in the real stratosphere (see McIntyre 1991 and references therein). The simple model put forth, in which a single band of uniform vorticity is located initially between compact polar vortices, certainly idealizes the actual vorticity distribution, to say nothing of its vertical structure. Yet it is plausible that the instability and roll-up of filamentary potential vorticity anomalies in stratified geophysical flows arises from fundamentally the same mechanism, since it is the potential vorticity distribution and the geometry containing the flow which essentially describe the instantaneous fluid motion and the interaction between its parts. Some new effects arising from the coupling between different layers in a stratified environment are examined by Waugh \& Dritschel (1991), though again the basic mechanism is the same. The results of this paper illustrate the important, destabilizing effect of spherical geometry and give a qualitative guide for the behaviour of filaments of potential vorticity strewn around a vortex following wave breaking. The polar distribution of vorticity in the atmospheres of rapidly rotating planets like the Earth singles out the equatorial region as the most likely place to form coherent vortices by way of filament instability, and it is a curious fact that this is where the large vortices on Jupiter and Neptune are located.

D.G.D. is supported by a grant from the UK Science and Engineering Research Council. L.M.P. acknowledges support from the Atmospheric Science Division of the US National Science Foundation, and is grateful to Dr M. E. McIntyre and his Research Group for their hospitality during his stay in Cambridge. The computations were carried out on the Amdahl VP1200 at the Manchester Computer Centre. The authors are grateful to Dr R. Saravanan for developing a method for shading the vorticity distribution in spherical contour dynamics calculations.

\section{Appendix A. Derivation of the governing equations in Lagrangian form}

In this appendix, we briefly review the elements of the derivation of equation (1) in the text. For a more complete account, see Dritschel (1988a or 1989b). The starting point is the relationship between stream function and vorticity,

$$
\nabla^{2} \psi=\omega,
$$

where here the Laplacian is restricted to the spherical surface. In the natural coordinates for the sphere, $z$ (sine of latitude) and $\phi$ (longitude), we have

$$
\nabla^{2} \psi=\frac{\partial}{\partial z}\left(\left(1-z^{2}\right) \frac{\partial \psi}{\partial z}\right)+\frac{1}{1-z^{2}} \frac{\partial^{2} \psi}{\partial \phi^{2}}
$$

The Green function needed for the inversion of $\psi$, i.e.

satisfies

$$
\begin{gathered}
\psi(z, \phi)=\iint G\left(z^{\prime}, \phi^{\prime} ; z, \phi\right) \omega\left(z^{\prime}, \phi^{\prime}\right) \mathrm{d} z^{\prime} \mathrm{d} \phi^{\prime}, \\
\nabla^{\prime 2} G=\frac{1}{4 \pi}\left(\delta\left(z^{\prime}-z\right) \delta\left(\phi^{\prime}-\phi\right)-1\right),
\end{gathered}
$$

the constant arising from the necessity that the integral of the Laplacian over a finite surface must vanish identically. The solution of this equation is elementary since, by 
symmetry of the surface, $G$ can only depend on the distance between two points. One can readily verify that

$$
G=\frac{1}{2 \pi} \log \left|x-x^{\prime}\right|,
$$

or the logarithm of the cord distance between two points (here $x=(r \cos \phi, r \sin \phi, z)$ with $r=\left(1-z^{2}\right)^{\frac{1}{2}}$, and likewise for $\left.x^{\prime}\right)$.

The next step of the derivation is to focus on the calculation of the axial velocity $w=\mathrm{d} z / \mathrm{d} t$; the other components can be obtained afterwards by symmetry. The reason why $z$ and $\phi$ are the natural coordinates for the sphere is because

$$
\frac{\mathrm{d} z}{\mathrm{~d} t}=\frac{\partial \psi}{\partial \phi}, \quad \frac{\mathrm{d} \phi}{\mathrm{d} t}=-\frac{\partial \psi}{\partial z}
$$

and $\mathrm{d} z \mathrm{~d} \phi$ is differential surface area. Using only the first of these equations, we have

$$
\frac{\mathrm{d} z}{\mathrm{~d} t}=\frac{\partial}{\partial \phi} \iint G\left(z^{\prime}, \phi^{\prime} ; z, \phi\right) \omega\left(z^{\prime}, \phi^{\prime}\right) \mathrm{d} z^{\prime} \mathrm{d} \phi^{\prime}=-\iint \frac{\partial G}{\partial \phi^{\prime}}\left(z^{\prime}, \phi^{\prime} ; z, \phi\right) \omega\left(z^{\prime}, \phi^{\prime}\right) \mathrm{d} z^{\prime} \mathrm{d} \phi^{\prime}
$$

since $G$ depends only on the angular difference $\phi^{\prime}-\phi$.

Now consider a single patch of vorticity, $\omega=$ constant in a region $R$ bounded by a contour $\mathscr{C}$. Define the inside of $\mathscr{C}$ to be the part of the sphere to be the left of $\mathscr{C}$. Then we can use Stoke's theorem to obtain

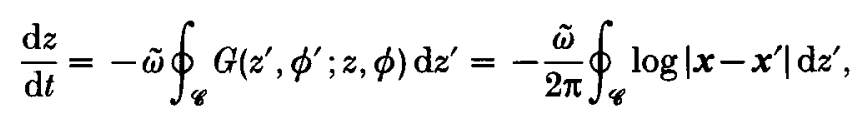

and hence by symmetry

$$
\frac{\mathrm{d} x}{\mathrm{~d} t}=-\frac{\tilde{\omega}}{2 \pi} \oint_{\mathscr{C}} \log \left|x-x^{\prime}\right| \mathrm{d} x^{\prime} .
$$

Here $\tilde{\omega}$ is the vorticity jump crossing into $\mathscr{C}$. It is related to interior and exterior vorticity by the obvious relation

$$
\tilde{\omega}=\omega_{\text {in }}-\omega_{\text {out }},
$$

but the constraint of zero total circulation (zero integral of Laplace's equation) provides a second relation,

$$
\omega_{\text {in }} A+\omega_{\text {out }}(4 \pi-A)=0,
$$

where $A$ is the area inside $\mathscr{C}$ (i.e. $A=\oint_{\mathscr{E}}(1-z) \mathrm{d} \phi$ ). Hence, given any one of $\tilde{\omega}, \omega_{\text {in }}$ or $\omega_{\text {out }}$, the other two are determined. The contour dynamical equations need only know about $\tilde{\omega}$ - the constraint of zero total circulation is automatically satisfied by construction. For later diagnostic purposes, for example producing plots of the vorticity like those shown in this paper, one can use the above relations to obtain the absolute vorticity anywhere on the surface.

\section{Appendix B. The dispersion relation in the thin-band limit}

In this appendix, we show the direct connection between the dispersion relation for a thin band and the result for a straight band in planar geometry, first obtained by Rayleigh (1887). The starting point is equation (17a) for a finite band in spherical geometry. In the limit where the band thickness tends to zero, the width of the band 
$\Delta$ must be much less than the mean radial position $r$ of the band, $\Delta / r \ll 1$. The width $\Delta$ is related to $z_{0 \mathrm{n}}-z_{0 \mathrm{~s}} \equiv \Delta z$ by

$$
\Delta \approx \Delta z / r \text {. }
$$

Letting $\tilde{\omega}$ be the vorticity jump into the band, we have $\tilde{\omega}_{\mathrm{s}}=-\tilde{\omega}_{\mathrm{n}}=\tilde{\omega}$ and so, from (2) and (3), $\omega_{\mathrm{s}}=\omega_{\mathrm{n}}=-\frac{1}{2} \tilde{\omega} \Delta z$. Using these values in (6), we obtain $\omega_{ \pm}$from $(17 b, c)$ as

$$
\omega_{+} \approx \frac{\tilde{\omega} z m \Delta z}{r^{2}} ; \quad \omega_{-} \approx-\tilde{\omega}+\frac{\tilde{\omega} m \Delta z}{r^{2}}
$$

If now we introduce the corresponding planar wavenumber $k$, related to the azimuthal wavenumber $m$ via $m=k r$, these expressions simplify to

$$
\omega_{+} \approx-\tilde{\omega} z k \Delta ; \quad \omega_{-} \approx \tilde{\omega}(1-k \Delta) \text {. }
$$

Finally, we turn to the term $S^{2 m}$ in $(17 a)$. With $S$ defined in (14), we substitute $z_{0 n}=z+\frac{1}{2} \Delta z$ and $z_{0 \mathrm{~s}}=z-\frac{1}{2} \Delta z$, and linearize in $\Delta z / r^{2}=\Delta / r$ to obtain

$$
S^{2 m} \approx\left(1-\frac{2 \Delta}{r}\right)^{m} \approx\left(1-\frac{2 k \Delta}{m}\right)^{m}
$$

Letting $m \rightarrow \infty$, in the expectation that instabilities will have wavelengths comparable to the band thickness, we obtain

$$
S^{2 m} \rightarrow \mathrm{e}^{-2 k \Delta} .
$$

Hence, $(17 a)$ reduces in the thin-band limit to

$$
\sigma=\frac{1}{2} \tilde{\omega}\left\{z k \Delta \pm\left[(1-k \Delta)^{2}-\mathrm{e}^{-2 k \Delta}\right]^{\frac{1}{2}}\right\} .
$$

The term involving the square root is identical to Rayleigh's dispersion relation for an infinite strip in planar geometry. The other, neutral term arises from the effects of spherical geometry, specifically from the residual background vorticity that must be present to ensure that the globally integrated vorticity must vanish (Appendix A). This term has no effect on stability.

\section{REFERENCES}

DrITSCHEL, D. G. 1986 The nonlinear evolution of rotating configurations of uniform vorticity. J. Fluid Mech. 172, 157-182.

Dritschel, D. G. 1988 a Contour dynamics/surgery on the sphere. J. Comput. Phys. 79, 477-483.

Dritschel, D. G. $1988 b$ Nonlinear stability bounds for inviscid, two-dimensional, parallel or circular flows with monotonic vorticity, and the analogous three-dimensional quasigeostrophic flows. J. Fluid Mech. 191, 575-581.

Dritschel, D. G. $1988 c$ The repeated filamentation of two-dimensional vorticity interfaces. J. Fluid Mech. 194, 511-547.

Dritschel, D. G. $1989 a$ On the stabilization of a two-dimensional vortex strip by adverse shear, J. Fluid Mech. 206, 193-221.

Dritschel, D. G. $1989 b$ Contour dynamics and contour surgery: numerical algorithms for extended, high-resolution modelling of vortex dynamics in two-dimensional, inviscid, incompressible flows. Computer Phys. Rep. 10, 77-146.

Dritschel, D. G. \& Legras, B. 1991 Vortex stripping. J. Fluid Mech. (submitted). (See also Dritschel, D. G. In Mathematical Aspects of Vortex Dynamics (ed. R. E. Caflisch) ch. 10. SIAM, 1989.)

Juckes, M. N. 1987 Studies of stratospheric dynamics. Ph.D. thesis, University of Cambridge, England. 
Juckes, M. N. \& McIntyre M. E. 1987 A high-resolution one-layer model of breaking planetary waves in the stratosphere. Nature 328, 590-596.

LAMв, H. H. 1932 Hydrodynamics. Dover.

Legras, B. \& Dritschel, D. G. 1991 A comparison of the contour surgery and pseudo-spectral methods. J. Comput. Phys. (submitted).

MoIntyre, M. E. 1991 Atmospheric dynamics: some fundamentals, with observational implications. In Proc. Intl School Phys. 'Enrico F'ermi', CXV Course (ed. J. C. Gille \& G. Visconti). North-Holland.

RAYLEIGH, LoRD 1887 On the stability, or instability, of certain fluid motions. Proc. Lond. Math. Soc. 11, 57-70. (Also in Rayleigh's Theory of Sound, Vol. 2, §367, Dover, 1945.)

WaUgh, D. W. 1991 Contour surgery simulations of a forced polar vortex, (in preparation).

Waugh, D. W. \& Dritscher, D. G. 1991 The stability of filamentary vorticity in twodimensional geophysical vortex-dynamics models. J. Fluid Mech. 231, 575-598. 